Introduction The National Health Service (NHS) is the biggest employer in the United Kingdom (UK). Depression and anxiety are the most common reasons for sickness absence in the NHS. As part of a trial feasibility study, we developed an intervention to facilitate an earlier return to work (RTW) in NHS staff absent with common mental health disorders (CMHD).

Methods We used iterative methodology, based on MRC guidance. Evidence was sought from systematic reviews, guidelines, and work known to the research team on the key components of the case-management (Stage 1). During Stage 2, the evidence from Stage 1 was mapped onto the proposed intervention together with input from international experts and key stakeholders.

Results Evidence suggests that an intervention based on a casemanagement model using a biopsychosocial approach could be cost-effective and lead to earlier RTW. In our study, specially trained occupational health nurses will deliver the intervention. Case-management will be conducted during regular consultations (every 2 to 4 weeks). Key components will include: identifying obstacles to RTW, collaborative problem solving based on cognitive behaviour principles focussing on work outcomes, work-focused goal setting, development of a RTW plan, and peer support to increase return to work self-efficacy. Work adjustments, work visits or therapeutic RTW will be considered. The case-manager will communicate with the line and human resources managers and treating healthcare professionals after each consultation. A bespoke information leaflet will be developed and given to line managers and workers emphasising the therapeutic importance of early RTW.

Discussion To our knowledge WB2W is the first intervention addressing RTW among UK healthcare staff with CMHDs. A key output from this research will be a complete specification of the intervention package including a manual for training the case managers and practical service information to guide the design of a randomised controlled trial.

\section{SHARPS INJURIES AMONG HEALTH CARE PERSONNEL IN A CHINESE HOSPITAL}

L Xiaoxia, B Adam*. University of Debrecen, Faculty of Public Health, Department of Preventive Medicine, Debrecen, Hungary

\subsection{6/oemed-2018-ICOHabstracts.982}

Introduction Sharps injury is a penetrating wound from a needle, scalpel, or another sharp object that may result in exposure to blood or other body fluids. Sharps injuries constitute a serious occupational health problem for health care personnel and can result in high direct and indirect costs for the health care facility. The aim of the study was to examine the prevalence and characteristics of sharps injuries among Chinese health care workers.

Methods A questionnaire survey was carried out in a Chinese hospital to collect demographic and occupational data, information on sharps injuries and their reporting. Multiple logistic regression was used to analyse the potential risk factors of sharps injuries, including gender, age, education level, position, department and perception of the safety culture.

Results The 5 year prevalence of sharps injuries was found $41 \%$ among the hospital personnel. Sex, age and education did not influence significantly sharps injuries $(p=0.798$, $\mathrm{p}=0.886$ and $\mathrm{p}=0.47$, respectively). However, the position of staff and especially the department where they work significantly correlated with sustaining such an accident
( $\mathrm{p}=0.025$ and $\mathrm{p}<0.0001$, respectively). $86 \%$ of accidents hurt fingers, $90 \%$ of injuries were sustained in inpatient units. Six sharp devices were responsible for nearly $95 \%$ of all injuries and $86 \%$ of them occurred when health care workers were using the devices. Association could not be described between sharps injuries and the perceived culture of safety. About three-fourth of sharps injuries were not reported.

Discussion A high prevalence rate of sharps injuries was observed in the studied hospital, the majority of injured workers were nurses. The rate varied significantly by department and position. The study found significant under-reporting. Adequate level of occupational health and safety for health care personnel can only be provided with efficient prevention from sharps injuries, which needs information on risk factors and a well-functioning reporting system.

\section{SURVEY OF RESPIRATORY PROTECTION PROGRAM IN IRANIAN HOSPITALS}

${ }^{1} \mathrm{M}$ Honarbakhsh, ${ }^{2} \mathrm{M}$ Jahangiri, ${ }^{2} \mathrm{H}$ Ghaem, ${ }^{3} \mathrm{P}$ Farhadi. ${ }^{1}$ Student Research Committee, School of Health, Shiraz University of Medical Sciences, Shiraz, Iran; ${ }^{2}$ School of Health, Shiraz University of Medical Sciences, Shiraz, Iran; ${ }^{3}$ Industrial Management Department, School of management and Accounting, Allameh Tabataba'i University, Tehran - Iran

\subsection{6/oemed-2018-ICOHabstracts.983}

Introduction In hospitals, Health Care Workers (HCWs) are exposed to a wide range of respiratory hazards, which requires using respiratory protection equipment and implementation of Respiratory Protection Program (RPP). The aim of this cross-sectional study was to investigate the level of RPP implementation in 36 educational hospitals of Fars province, Iran.

Methods A Self-made checklist including nine components of RPP standard were prepared and completed by the occupational hygienists in hospitals. Fuzzy Analytical Hierarchy Process (FAHP) was used to determine the weight coefficient of RPP components. Finally, Respiratory Protection Program Index (RPPI) was developed to calculate the compliance degree of RPP.

Results The results showed that RPP was not fully implemented in the studied hospitals and the highest and lowest RPPI scores were related to training and fit testing, respectively.

Conclusion Effective protection of HCWs against respiratory hazards requires the full implementation of respiratory protection program in the hospitals. This program must be include evaluation of respiratory hazards, appropriate selection and maintenance of respirators, medical evaluation, fit testing, employee training record keeping, and program evaluation. However, the largest gaps in the RPP elements were discovered in the components of fit testing and medical evaluation which requires significant attempts to promote the situation of RPP in the studied hospital.

\section{NATIONAL HEPATITIS B VACCINATION POLICIES FOR HEALTHCARE WORKERS IN MEMBER-STATES OF THE EUROPEAN UNION: AN UPDATED OVERVIEW}

\footnotetext{
${ }^{1,2} \mathrm{~A}$ de Schryver*, ${ }^{2} \mathrm{~T}$ Lambaerts, ${ }^{2} \mathrm{~N}$ Lammertyn. ${ }^{1}$ University of Antwerpen, Epidemiology and Social Medicine, Antwerpen, Belgium; ${ }^{2}$ IDEWE Occupational Health Services, Leuven, Belgium
}

10.1136/oemed-2018-ICOHabstracts.984 